\title{
Construction of Variable-Stepsize Multistep Formulas
}

\author{
By Robert D. Skeel*
}

\begin{abstract}
A systematic way of extending a general fixed-stepsize multistep formula to a minimum storage variable-stepsize formula has been discovered that encompasses fixed-coefficient (interpolatory), variable-coefficient (variable step), and fixed leading coefficient as special cases. In particular, it is shown that the "interpolatory" stepsize changing technique of Nordsieck leads to a truly variable-stepsize multistep formula (which has implications for local error estimation and formula changing), and it is shown that the "variable-step" stepsize changing technique applicable to the Adams and backward-differentiation formulas has a reasonable generalization to the general multistep formula. In fact, it is shown how to construct a variable-order family of variable-coefficient formulas. Finally, it is observed that the first Dahlquist barrier does not apply to adaptable multistep methods if storage rather than stepnumber is the key consideration.
\end{abstract}

1. Introduction. Multistep methods have been the most successful numerical methods for solving initial-value problems in ordinary differential equations. The selection of a particular formula is often based on a theoretical analysis of fixedstepsize formulas, and yet implementation normally requires the use of a variablestepsize formula. The question of how to extend a formula to variable stepsize is the primary topic of this paper. Existing techniques for varying stepsize are studied, revealing interesting relationships and useful generalizations. At the same time, the results in Skeel [19] on the equivalence between multivalue methods and multistep methods are extended to variable stepsize. There are techniques for varying the stepsize other than the use of variable-stepsize formulas, and these are included in the survey of Krogh [13].

The question of how to extend a formula to variable stepsize has confronted a number of researchers. For example, Sand [15, p. 8] states:

Although there exist natural extensions of the two most common classes of fixed-step formulas to variable step-sizes, viz. the Adams LMF's and the BDF's... there exists no unique, and in general, no natural, variable-step version of a fixed-step formula.

The natural extensions referred to here are implemented in such codes as EPISODE (Byrne and Hindmarsh [1]) and ODE/DE/STEP, INTRP (Shampine and Gordon [17]), and have been named "variable-coefficient" formulas by Jackson and SacksDavis [11]. In Section 5 of this paper we present a systematic way of extending fixed-stepsize formulas to variable-stepsize formulas of which the natural variablecoefficient Adams and backward-differentiation formulas are particular cases. These

Received September 21, 1984; revised January 3, 1986.

1980 Mathematics Subject Classification. Primary 65L05.

Key words and phrases. Multistep formula, multistep method, multivalue method, variable stepsize, variable order.

*Work partially supported by the National Science Foundation under grant MCS 8107046 and by the U.S. Department of Energy under grant DEAC0276ERO2383. 
variable-coefficient formulas have attractive properties, that taken together are not possessed by other variable-stepsize extensions. They interpolate past data in some generalized sense at previous meshpoints, which suggests superior stability properties. Also, they require a minimum of storage.

The popular integrator LSODE (Hindmarsh [10]) does not use variable-coefficient formulas but rather "fixed-step interpolation" formulas based on an idea due to Nordsieck [14] and popularized by Gear [8]. This technique for varying the stepsize is applicable to general fixed-stepsize formulas. The precise nature of this type of variable-stepsize method has been somewhat of a mystery, but if there is a prevailing view, then it is most clearly expressed by Jackson and Sacks-Davis [11]:

If, in a fixed coefficient implementation, the stepsize is changed at every step, then the corrector equation... may be expressed in the form

$$
\alpha_{0} y_{n}+\sum_{j=1}^{n} \tilde{\alpha}_{n, j} y_{n-j}+h_{n} f_{n}+h_{n} \sum_{j=1}^{n-2} \tilde{\beta}_{n, j} f_{n-j}=0,
$$

provided, as well, that the order $k$ is always kept greater than two. Thus the solution at $t_{n}$ may depend on all of the previously computed values. Consequently, eq. (2.18) is not a "local formula" as are eqs. (2.6), (2.13), and (2.15). This gives one an intuitive understanding for the cause of the numerical instability of fixed coefficient formulas with respect to step-size changes

These comments are specific to the backward-differentiation formulas; presumably they should apply also to other linear multistep formulas if the $n-2$ in the limit of summation is changed to $n$. In Section 5 of this paper we show that the situation is not so bad, that fixed-coefficient formulas are true variable-stepsize formulas, meaning that the upper summation limits in (2.18) can be replaced by the stepnumber $k$ and the formula coefficients depend on only the last $k-2$ stepsize ratios. We expect that this information could be very important for the construction of local error estimates having a sound theoretical basis. There is little reason to believe that the local error estimators used in current implementations are asymptotically correct, even (for nonstiff problems) with slowly varying stepsize.

The variable-coefficient and fixed-coefficient extensions are related even more closely than has already been suggested. They are both specializations of a more general technique for variable-stepsize extension discussed in Section 2. This generic technique for extending formulas to variable stepsize is characterized by the fact that it creates methods having minimal storage requirements.

A fixed-stepsize linear $k$-step formula, $k \geqslant 2$, for the ODE $y^{\prime}(t)=f(t, y(t))$ determines approximations $y_{n}$ and $y_{n}^{\prime}$ to the solution and its derivative at a point $t_{n}$ from the two equations

$$
\begin{gathered}
\alpha_{0} y_{n}+\alpha_{1} y_{n-1}+\cdots+\alpha_{k} y_{n-k}=h\left(\beta_{0} y_{n}^{\prime}+\beta_{1} y_{n-1}^{\prime}+\cdots+\beta_{k} y_{n-k}^{\prime}\right), \\
y_{n}^{\prime}=f\left(t_{n}, y_{n}\right),
\end{gathered}
$$

assuming that approximations $y_{n-j}, y_{n-j}^{\prime}$ are available at previous meshpoints $t_{n-j}:=t_{n}-j h, j=1(1) k$. It is not necessary for our purposes that Eq. (1.1b) be satisfied exactly. We assume that $\alpha_{0} \neq 0$ and that the polynomials

$$
\rho(\xi):=\alpha_{0} \xi^{k}+\alpha_{1} \xi^{k-1}+\cdots+\alpha_{k} \text { and } \sigma(\xi):=\beta_{0} \xi^{k}+\beta_{1} \xi^{k-1}+\cdots+\beta_{k}
$$

have no common factors, which is equivalent to the nonexistence of an "equivalent" formula of lower stepnumber. From the latter assumption it follows that $\alpha_{k}^{2}+\beta_{k}^{2}>$ 0 . Assume the formula is of order $\geqslant q$ where $k \leqslant q \leqslant 2 k-1$, which for linear 
multistep methods is equivalent to requiring that the formula be exact if $y(t)$ is a polynomial of degree $\leqslant q$. The cases $q=k-1$ and $q=2 k$ are excluded because they require special treatment. The order of the (fixed-stepsize) formula may exceed $q$, but we shall seek a variable-stepsize extension of order $q$ only.

For nonstiff problems, stability considerations (strong stability) constrain the order to be $\leqslant k+1$. The archetypal nonstiff formula is the Adams-Moulton formula (AMF)

$$
y_{n}-y_{n-1}=h\left(\beta_{0} y_{n}^{\prime}+\beta_{1} y_{n-1}^{\prime}+\cdots+\beta_{k} y_{n-k}^{\prime}\right),
$$

which is of order $k+1$. For stiff problems, stability considerations ( $A(\alpha)$-stability) constrain the order to be $\leqslant k$ (except for the trapezoidal rule), and the archetypal stiff formula is the backward-differentiation formula (BDF)

$$
\alpha_{0} y_{n}+\alpha_{1} y_{n-1}+\cdots+\alpha_{k} y_{n-k}=h y_{n}^{\prime},
$$

which is of order $k$. Both sets of formulas are attractive because of their simple derivation, but it is difficult to believe that they are the best for all special-purpose and general-purpose codes. We note that other formulas have been derived by many authors and that some of these have been used to solve practical problems, for example, the $K$-method of Kregel and Heimerl [12] used at the U.S. Army Ballistic Research Laboratory.

For various practical reasons (error control, efficiency, starting, solution of nonlinear systems) we want to use variable stepsize $h_{n}:=t_{n}-t_{n-1}$. For a given fixed-stepsize formula we want a variable-stepsize extension

$$
\sum_{j=0}^{k} \alpha_{j n} y_{n-j}=\beta_{0 n} h_{n} y_{n}^{\prime}+\sum_{j=1}^{k} \beta_{j n} h_{n-j+1} y_{n-j}^{\prime} .
$$

There are a number of restrictions that are clearly desirable. The coefficients $\alpha_{j n}, \beta_{j n}$ should depend only on $t_{n}, t_{n-1}, \ldots, t_{n-k}$, and, in fact, they ought to be rational functions of the stepsize ratios $r_{n}, r_{n-1}, \ldots, r_{n-k+2}$. Here, $r_{n}:=h_{n} / h_{n-1}$. We will not require that the coefficients exist for all possible combinations of stepsize ratios, although this is desirable. It goes without saying that the variable-stepsize coefficients should be equal to the fixed-stepsize coefficients when the stepsize ratios are all one. The normalization

$$
\alpha_{0 n}=\alpha_{0}
$$

will be assumed. Any other normalization may not always be applicable; for example, the more natural normalization $\sum \beta=1$ sometimes fails for the $f$ variant of the fixed leading coefficient formulas of Jackson and Sacks-Davis [11]. Requiring that the formula be exact for polynomials of degree at most $q$ imposes another $q+1$ linear conditions on the coefficients. However, we need yet another $2 k-q$ conditions, which is the main topic of this paper.

Note. Our use of $h_{n-j+1}$ instead of $h_{n}$ as a coefficient for $y_{n-j}^{\prime}, j \geqslant 2$, is unusual but for our purposes more convenient.

For the $k$-step AMF we need $k-1$ auxiliary conditions and the most popular choice (variable coefficient) is

$$
\alpha_{2 n}=\alpha_{3 n}=\cdots=\alpha_{k n}=0 .
$$


For the $k$-step BDF we need $k$ additional conditions and a popular choice (variable coefficient again) is

$$
\beta_{1 n}=\beta_{2 n}=\cdots=\beta_{k n}=0 .
$$

One motivation for these conditions, emphasized in the paper of Dill and Gear [7], is to keep down the number of saved values (more specifically, the number of values that must be saved between steps). For this variable-stepsize extension of AMF it is clear that only the $k+1$ values $y_{n-1}, y_{n-1}^{\prime}, \ldots, y_{n-k}^{\prime}$, are needed to determine $y_{n}$ and $y_{n}^{\prime}$, and for the BDF only $k$ values are needed. However, this property is not unique to these choices of coefficients. It is shown in Skeel [19] that any fixed-stepsize $k$-step method requires only $k$ values to be saved in order to advance the solution (and this is without reevaluating the right-hand side $f(t, y)$ ). This also follows from the equivalence between linear multistep and one-leg methods discovered by Dahlquist [3], as well as the modifier polynomial formalism of Wallace and Gupta [21]. In practice we want to use a predictor of order $q$ at least, so that we get a good initial guess for the nonlinear equation solver, so that the Milne device can be used to estimate local errors, and so that interpolation to off-mesh points can be performed with an error of only $O\left(h^{q+1}\right)$. Thus, we should save $q+1$ values.

The same trick used to economize on storage for fixed-stepsize methods also works for variable-stepsize methods. Let us illustrate this with the 2-step AdamsBashforth formula by choosing the $2 k-q=2$ auxiliary conditions plus the one normalization condition to be

$$
\beta_{0 n}=0, \quad \beta_{1 n}=\frac{3}{2}, \quad \beta_{2 n}=-r_{n} / 2 .
$$

Requiring that the formula be exact for second-degree polynomials yields

$$
\alpha_{0 n}=2 /\left(1+r_{n}\right), \quad \alpha_{1 n}=r_{n}-2, \quad \alpha_{2 n}=r_{n}\left(1-r_{n}\right) /\left(1+r_{n}\right) .
$$

If we are given the three values

$$
y_{n-1}, \quad y_{n-1}^{\prime}, \quad \tilde{s}_{n-2}^{0}:=-\alpha_{2 n} y_{n-2}+\beta_{2 n} h_{n-1} y_{n-2}^{\prime},
$$

we can determine $y_{n}, y_{n}^{\prime}, \tilde{s}_{n-1}^{0}$ from

$$
\begin{aligned}
& \tilde{s}_{n-1}^{0}=-\alpha_{2, n+1} y_{n-1}+\beta_{2, n+1} h_{n} y_{n-1}^{\prime}, \\
& \alpha_{0 n} y_{n}+\alpha_{1 n} y_{n-1}=\beta_{1 n} h_{n} y_{n-1}^{\prime}+\tilde{s}_{n-2}^{0}, \\
& y_{n}^{\prime}=f\left(t_{n}, y_{n}\right) .
\end{aligned}
$$

There is a disturbing feature of this algorithm, that the reader may have noticed. It concerns the computation of $\tilde{s}_{n-1}^{0}$ at time $t_{n}$ and the fact that this requires knowing the value of $t_{n+1}$. For adaptive methods, the value of $t_{n+1}$ is determined, by trial and error, only after completely advancing to $t_{n}$.

The failure of our algorithm in this example is probably typical. For a second example, consider the constant- $\rho$ variable-stepsize extension proposed by Gear and Watanabe [9], in which we require $\alpha_{j n}=\alpha_{j}, 0 \leqslant j \leqslant k$, so that the variable-stepsize formula inherits the 0 -stability properties of the fixed-stepsize formula. (This actually constitutes only $k-1$ auxiliary conditions because a normalization is included and the formula is already exact for constant polynomials.) The result of applying the constant- $\rho$ variable-stepsize extension to the third-order formula with

$$
\alpha_{0}=\frac{3}{2}, \quad \alpha_{1}=-2, \quad \alpha_{2}=\frac{1}{2},
$$


has

$$
\beta_{0 n}=\frac{6 r^{3}+9 r^{2}+1}{12 r^{2}(r+1)}, \quad \beta_{1 n}=\frac{3 r^{3}+9 r^{2}-3 r-1}{12 r^{2}}, \quad \beta_{2 n}=-\frac{3 r^{3}+3 r+2}{12(r+1)},
$$

where $r=r_{n}$.

Therefore, in our search for auxiliary conditions we impose one more restriction:

The $2 k-q$ auxiliary conditions should be such that only $q+1$ values need be saved without knowing future meshpoints.

Such methods we call adaptable $(q+1)$-value methods. At the beginning of Section 2 we give generic auxiliary conditions that are sufficient and seem to be necessary in order that the storage be minimal without predetermined meshpoints.

It is convenient to introduce $m:=(q+1)-k$ and to use $m$ rather than $q$ in our discussion. Thus we are seeking $k-m+1$ auxiliary conditions for a $(k+m)$-value method of order $k+m-1$. The assumption $k \leqslant q \leqslant 2 k-1$ implies $1 \leqslant m \leqslant k$. Typically, $m=1$ for a stiff formula, and $m=2$ for a nonstiff formula.

An auxiliary condition proposed by Jackson and Sacks-Davis [11] is to fix $\beta_{0 n}=\beta_{0}$. Such fixed leading coefficient variable-stepsize formulas have certain practical advantages for stiff ODEs, and these formulas are considered in Section 6. On the other hand, there is some suggestion, namely, the equivalence of fixed leading coefficient to fixed-coefficient for the second-order BDF, that variable leading coefficient formulas have better stability properties.

If the meshpoints were known in advance, we could express the unique variablestepsize $k$-step formula of order $2 k$ in such a way that only $k$ values need to be saved. However, if the new meshpoint $t_{n}$ is not known in advance, we cannot expect the order of a $q$-value method to exceed $q$, because there are only $q+1$ items of information available to determine the solution at $t_{n}$ : the $k$ saved values plus the differential equation at $t_{n}$. (Consider, in particular, the problem $y^{\prime}=f(t)$.) Moreover, the $q$ th order Adams-Moulton formula can be expressed as an adaptable $q$-value method, and thus there exist strongly 0 -stable $q$-value methods of optimal order. Therefore, the first Dahlquist [2] barrier does not exist for adaptable variable-stepsize formulas, if we consider the number of saved values rather than the stepnumber of the formula. In Section 3 we construct an adaptable $q$ th-order $q$-value variable-stepsize extension for any $q$ th-order fixed-stepsize formula with stepnumber $<q$.

In the fixed-stepsize case, Skeel [19] shows how to formulate a $(k+m)$-value method using a polynomial $p_{n}(t)$ that interpolates $k+m$ values as follows:

$$
\begin{gathered}
p_{n}\left(t_{n-j}\right)=y_{n-j}, \quad j=0(1) m-1, \\
p_{n}^{\prime}\left(t_{n-j}\right)=y_{n-j}^{\prime}, \quad j=0(1) m-1, \\
\sum_{i=0}^{j}\left(-\alpha_{k-j+i} p_{n}\left(t_{n-m-i}\right)+h \beta_{k-j+i} p_{n}^{\prime}\left(t_{n-m-i}\right)\right) \\
=\sum_{i=0}^{j}\left(-\alpha_{k-j+i} y_{n-m-i}+h \beta_{k-j+i} y_{n-m-i}^{\prime}\right), \quad j=0(1) k-m-1 .
\end{gathered}
$$

In advancing from $p_{n-1}(t)$ to $p_{n}(t)$ we obtain one new item of information, namely, the derivative value at $t_{n}$. (This is obviously the case for the ODE $y^{\prime}=f(t)$.) Hence, the polynomials $p_{n-1}(t)$ and $p_{n}(t)$ interpolate nearly the same data and so we 
expect the difference to be especially simple. In fact,

$$
p_{n}(t)=p_{n-1}(t)+\frac{1}{\alpha_{0}}\left(y_{n}^{\prime}-p_{n-1}^{\prime}\left(t_{n}\right)\right) \Lambda\left(\frac{t-t_{n}}{h}\right),
$$

where the modifier polynomial $\Lambda(x)$ is determined by the formula through the conditions

$$
\begin{aligned}
\Lambda(-j) & = \begin{cases}\beta_{0}, & j=0, \\
0, & j=1(1) m-1,\end{cases} \\
\Lambda^{\prime}(-j) & = \begin{cases}\alpha_{0}, & j=0, \\
0, & j=1(1) m-1,\end{cases} \\
\sum_{i=0}^{j}\left\{-\alpha_{k-j+i} \Lambda(-m-i)+\beta_{k-j+1} \Lambda^{\prime}(-m-i)\right\}=0, & j=0(1) k-m-1 .
\end{aligned}
$$

It is shown (Skeel [19, Corollary to Theorem 2.1]) that these conditions uniquely determine $\Lambda(x)$. As an example, the $k$-step BDF has

$$
\Lambda(x)=\left(\begin{array}{c}
x+k \\
k
\end{array}\right) \text {. }
$$

The modifier polynomial was discovered in special cases by Descloux [6] and Byrne and Hindmarsh [1] and in general by Wallace and Gupta [21] and Skeel [19].

Equation (1.2) is an alternative way of expressing a linear multistep formula and has a number of advantages. First, a $k$ th degree modifier polynomial $\Lambda(x)$ is a convenient parameterization of a linear $k$-step formula because the number of free coefficients after a normalization (such as $\Lambda^{\prime}(0)=1$ or $\Lambda^{(k)}(0)=1$ which corresponds to $\alpha_{0}=1$ or $\sum \beta=1$, respectively) exactly equals the $k$ degrees of freedom in a normalized, linear multistep formula of order $\geqslant k$ and stepnumber $\leqslant k$. Second, the minimum storage implementation is obvious from (1.2). The coefficients are easily calculated from $\Lambda(x)$; for example, a divided-difference implementation would use divided differences of $\Lambda\left(\left(t-t_{n}\right) / h\right)$. Third, there is a built-in predictor. Fourth, there is a built-in interpolation to off-mesh points. However, there is a disadvantage to the form (1.2), and that is that it seems to be less convenient for the analysis of stability and accuracy.

The modifier polynomial $\Lambda(x)$ is used in Section 5 to give the auxiliary conditions for the variable-coefficient extension. There is another more direct way of constructing the variable-coefficient formula, which we give here:

For the values $y_{n-j}$ and $y_{n-j}^{\prime}, j=0(1) m-1$,

$$
\begin{aligned}
s_{n-m}^{j}:=\sum_{i=0}^{j}\left\{-\alpha_{k-j+i} y_{n-m-i}+\beta_{k-j+i} h_{n-m-i+1} y_{n-m-i}^{\prime}\right\} & \\
j & =0(1) k-m,
\end{aligned}
$$

we determine $k+m$ coefficients $\alpha_{j n}, \beta_{j n}, \gamma_{j n}$, such that the formula

$$
\alpha_{0} y_{n}-\beta_{0 n} h_{n} y_{n}^{\prime}=\sum_{j=1}^{m-1}\left\{-\alpha_{j n} y_{n-j}+\beta_{j n} h_{n-j+1} y_{n-j}^{\prime}\right\}+\sum_{j=m}^{k} \gamma_{j n} s_{n-m}^{j-m}
$$

is exact for polynomials of degree $\leqslant k+m-1$. 
The auxiliary conditions for the fixed-coefficient case are given in Section 7 in terms of the modifier polynomial $\Lambda(x)$. But again there is another, more direct way of describing this method. Assume we are given

$$
y_{n-1,-j} \simeq y\left(t_{n-1}-j h_{n-1}\right), \quad y_{n-1,-j}^{\prime} \simeq y^{\prime}\left(t_{n-1}-j h_{n-1}\right)
$$

for $j=0(1) k-1$. Let $p_{n-1}(t)$ be the unique polynomial of degree at most $k+m$ - 1 which interpolates (as before) the following equally spaced data:

$$
\begin{gathered}
y_{n-1,-j}, y_{n-1,-j}^{\prime}, \quad j=0(1) m-1, \\
\sum_{i=0}^{j}\left\{-\alpha_{k-j+i} y_{n-1,-m-i}+h_{n-1} \beta_{k-j+i} y_{n-1,-m-i}^{\prime}\right\}, \quad j=0(1) k-m-1 .
\end{gathered}
$$

Then, evaluate $p_{n-1}(t)$ and its derivative at the $k$ points from $t_{n-1}$ backwards with uniform spacing $h_{n}$,

$$
y_{n,-j}=p_{n-1}\left(t_{n}-j h_{n}\right), \quad y_{n,-j}^{\prime}=p_{n-1}^{\prime}\left(t_{n}-j h_{n}\right),
$$

$j=1(1) k$, and apply the fixed-stepsize formula (1.1) to those values in order to obtain $y_{n}$ and $y_{n}^{\prime}$.

As previously stated, a fixed-coefficient method can be expressed as a true variable-stepsize formula, and as an example, the fixed-coefficient second-order BDF has the form

$$
\frac{3}{2} y_{n}-\frac{3+r_{n}^{2}}{2} y_{n-1}+\frac{r_{n}^{2}}{2} y_{n-2}=h_{n} y_{n}^{\prime}+\frac{1-r_{n}}{2} h_{n} y_{n-1}^{\prime} .
$$

We have been considering fixed-formula methods only. For variable-formula methods the idea of the number of saved values still applies, although this number might have to be greater because of the possibility of changing formulas. Clearly, the various formulas ought to be related in such a way that the number of saved values is low. More specifically, if we want a family of variable-coefficient formulas indexed by $k=m(1) K$, such that the $k$ th formula has stepnumber $k$ and order $k+m-1$, then we should begin by choosing the $K$ th formula to be whatever we like, and for the $k$ th formula, $k=m(1) K-1$, we should use the unique formula of order $k+m-1$ that relates the values

$$
y_{n-j}, y_{n-j}^{\prime}, \quad j=0(1) m-1, \quad s_{n-m}^{j}, \quad j=0(1) k-m,
$$

where the latter are defined using the coefficients of the $K$ th formula. This is further discussed in Section 4 in greater generality.

Sections 2 through 7 appear in the supplements section at the end of this issue.

We conclude this section by offering some opinions about the various ideas sparked by Nordsieck [14] and pursued by a number of authors, especially Gear [8]. These ideas have had a profound practical impact but have otherwise been resisted by most researchers in numerical ODEs. As shown in this and previous papers, all interesting multivalue methods can be expressed as genuine multistep methods, although for blended methods this can be complicated [20]. The multistep form seems to be best for the analysis of errors and of stability. Nonetheless both the "number of saved values" and the "modifier polynomial" seem to be useful ideas worth retaining.

There is significant experimental and theoretical evidence in the case of Adams and backward-differentiation formulas that variable-coefficient methods are stable for a greater variety of stepsize sequences than are the fixed-coefficient methods. 
Stability is a very important consideration, more so than the complexity of coefficient calculation (or the representation of saved values), and for this reason the fixed-coefficient schemes should be abandoned in favor of either variable-coefficient or fixed leading coefficient schemes, which, in this paper, have been defined for general linear multistep formulas. Even these may have inadequate stability properties compared to the second-order 2-step one-leg formulas of Dahlquist, Liniger, and Nevanlinna [5].

Department of Computer Science

University of Illinois at Urbana-Champaign

240 Digital Computer Laboratory

1304 W. Springfield Avenue

Urbana, Illinois 61801

1. G. D. Byrne \& A. C. Hindmarsh, "A polyalgorithm for the numerical solution of ordinary differential equations," ACM Trans. Math. Software, v. 1, 1975, pp. 71-96.

2. G. G. DAHLQUIST, "Numerical integration of ordinary differential equations," Math. Scand., v. 4, 1956, pp. 33-50.

3. G. G. Dahlquist, On Stability and Error Analysis for Stiff Non-linear Problems, Part 1, Report TRITA-NA-7508, Dept. of Computer Sci., Royal Inst. of Technology, Stockholm, 1975.

4. G. G. Dahlquist \& A. BJORCK, Numerical Methods (transl. by N. Anderson), Prentice-Hall, Englewood Cliffs, N. J., 1975.

5. G. G. Dahlquist, W. Liniger \& O. Nevanlinna, "Stability of two-step methods for variable integration steps," SIAM J. Numer. Anal., v. 20, 1983, pp. 1071-1085.

6. J. Descloux, A Note on a Paper by A. Nordsieck, Report \#131, Dept. of Computer Sci., Univ. of Illinois, Urbana-Champaign, 1963.

7. C. Dill \& C. W. Gear, "A graphical search for stiffly stable methods for ordinary differential equations," J. Assoc. Comput. Mach., v. 18, 1971, pp. 75-79.

8. C. W. Gear, Numerical Initial Value Problems in Ordinary Differential Equations, Prentice-Hall, Englewood Cliffs, N. J., 1971.

9. C. W. Gear \& D. S. Watanabe, "Stability and convergence of variable order multistep methods," SIAM J. Numer. Anal., v. 11, 1974, pp. 1044-1058.

10. A. Hindmarsh, Documentation for LSODE, Math. \& Stats. Section L-300, Lawrence Livermore Laboratory, Livermore, Calif., 1980.

11. K. R. JACKSON \& R. SACKS-DAvis, "An alternative implementation of variable step-size multistep formulas for stiff ODEs," ACM Trans. Math. Software, v. 6, 1980, pp. 295-318.

12. M. D. Kregel \& J. M. Heimerl, Comments on the Solution of Coupled Stiff Differential Equations, Proc. of the 1977 Army Numerical Analysis and Computers Conference, Report No. 77-3, U. S. Army Research Office, Research Triangle Park, N. C., 1977, pp. 553-563.

13. F. T. KroGH, "Algorithms for changing the step size," SIAM J. Numer. Anal., v. 10, 1973, pp. 949-965.

14. A. NoRDSIECK, "On numerical integration of ordinary differential equations," Math. Comp., v. 16, 1962, pp. 22-49.

15. J. SAND, Stability and Boundedness Results for Variable-Step Variable-Formula Methoc's, Report TRITA-NA-8219, Dept. of Numer. Anal. and Computer Sci., Royal Inst. of Technology, Stockholm, 1982.

16. L. F. Shampine, How to Live with a Reasonable ODE Code (DIFSUB) until a Good One Arrives, manuscript, ca 1974.

17. L. F. Shampine \& M. K. Gordon, Computer Solution of Ordinary Differential Equations, Freeman, San Francisco, 1975.

18. R. D. SkeEL, Convergence of Multivalue Methods for Solving Ordinary Differential Equations, Report TR73-16, Dept. of Computing Sci., Univ. of Alberta, Edmonton, 1973.

19. R. D. SKEEL, “Equivalent forms of multistep formulas," Math. Comp., v. 33, 1979, pp. 1229-1250; Corrigendum, ibid., v. 47, 1986, p. 769.

20. R. D. SkeEL \& T. V. VU, "Note on blended linear multistep methods." (submitted.)

21. C. S. Wallace \& G. K. Gupta, "General linear multistep methods to solve ordinary differential equations," Austral. Comput. J., v. 5, 1973, pp. 62-69. 


\title{
Supplement to \\ Construction of Variable-Stepsize Multistep Formulas
}

\author{
By Robert D. Skeel
}

\section{Generic Auxiliary Conditions}

We are seeking $k-m+1$ auxiliary conditions on variable stepsize formula coefficients so that only $k+m$ values need be saved between steps without knowing future meshpoints. It is believed that such conditions can always be put in the form

$$
\left[-\alpha_{1 n}, \ldots, \beta_{k n}\right] E^{j} \mathbf{g}_{n-j}=0, \quad j=0(1) k-m,
$$

where $\left[-\alpha_{1 n}, \ldots, \beta_{k n}\right]$ is an abbreviation for $\left[-\alpha_{1 n}, \beta_{1 n}, \ldots,-\alpha_{k n}, \beta_{k n}\right]$,

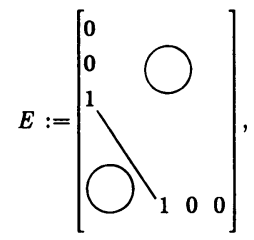

$$
\begin{aligned}
& \mathbf{g}_{n}:=\left[0, \ldots, 0, c_{m n}, d_{m n}, \ldots, c_{k n}, d_{k n}\right]^{T}, \\
& c_{j n}:=\text { function }\left(r_{n}, r_{n-1}, \ldots, r_{n-j+2}\right),
\end{aligned}
$$

and

$$
d_{j n}:=\text { function }\left(r_{n}, r_{n-1}, \ldots, r_{n-j+2}\right) .
$$

We assume that these functions are rational functions that are well defined for all positive stepsize ratios and that the coefficients $\alpha_{j n}$ and $\beta_{j n}$ are uniquely determined to be $\alpha_{j}$ and $\beta$, when the stepsize ratios are all one. The complete set of $2 k+2$ conditions that determine the formula coefficients will be called the v.l.c. conditions.

The mere existence of $c_{j n}$ and $d_{j n}$ such that (2.1) holds does impose substantial restrictions on the coefficients of the variable stepsize formula. For example, from (2.1) with $j=k-m$ we get

$$
-c_{m, n-k+m} \alpha_{k n}+d_{m, n-k+m} \beta_{k n}=0 \text {. }
$$

Our assumptions imply that for uniform stepsize

$$
-c_{m} \alpha_{k}+d_{m} \beta_{k}=0
$$

and

$$
c_{m}^{2}+d_{m}^{2}>0
$$

Taking $d_{m} \alpha_{k}+c_{m} \beta_{k}$ times (2.2) and subtracting $d_{m, n-k+m} \alpha_{k n}+c_{m, n-k+m} \beta_{k n}$ times (2.3) yields

$$
\frac{-\beta_{k} \alpha_{k n}+\alpha_{k} \beta_{k n}}{\alpha_{k} \alpha_{k n}+\beta_{k} \beta_{k n}}=\frac{d_{m} c_{m, n-k+m}-c_{m} d_{m, n-k+m}}{c_{m} c_{m, n-k+m}+d_{m} d_{m, n-k+m}},
$$

which implies that the left-hand side must be a rational function of $r_{n-k+m}, r_{n-k+m-1}, \ldots, r_{n-k+2}$ only. If $m<k$, this is a real restriction, not satisfied by the two examples of Section 1 . In fact, if $m=1$, the numerator of the left-hand side must be identically sero, and as a corollary we get that $\alpha_{k n} \equiv 0$ if $\alpha_{k}=0$ and $\beta_{k n} \equiv 0$ if $\beta_{k}=0$. 

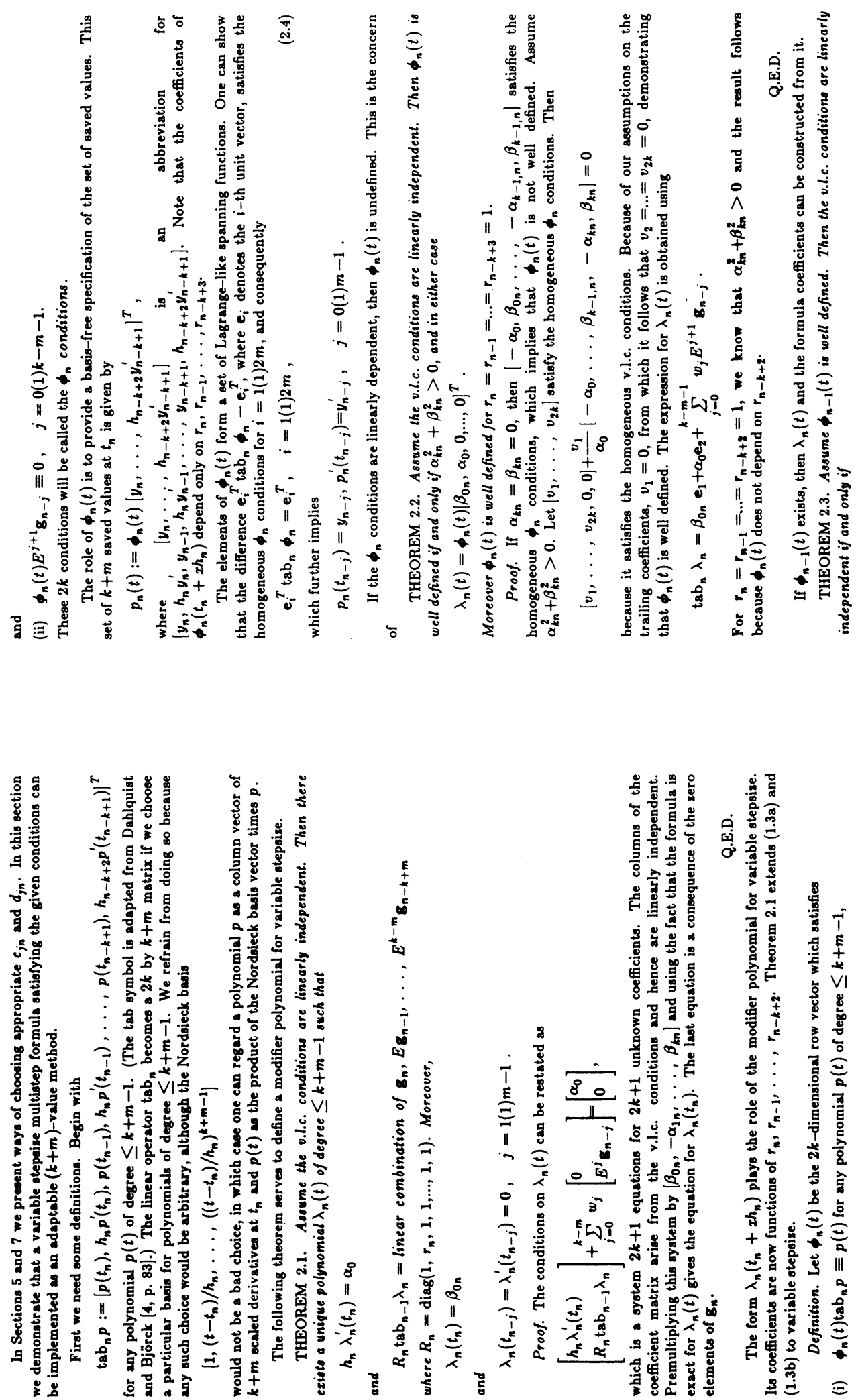

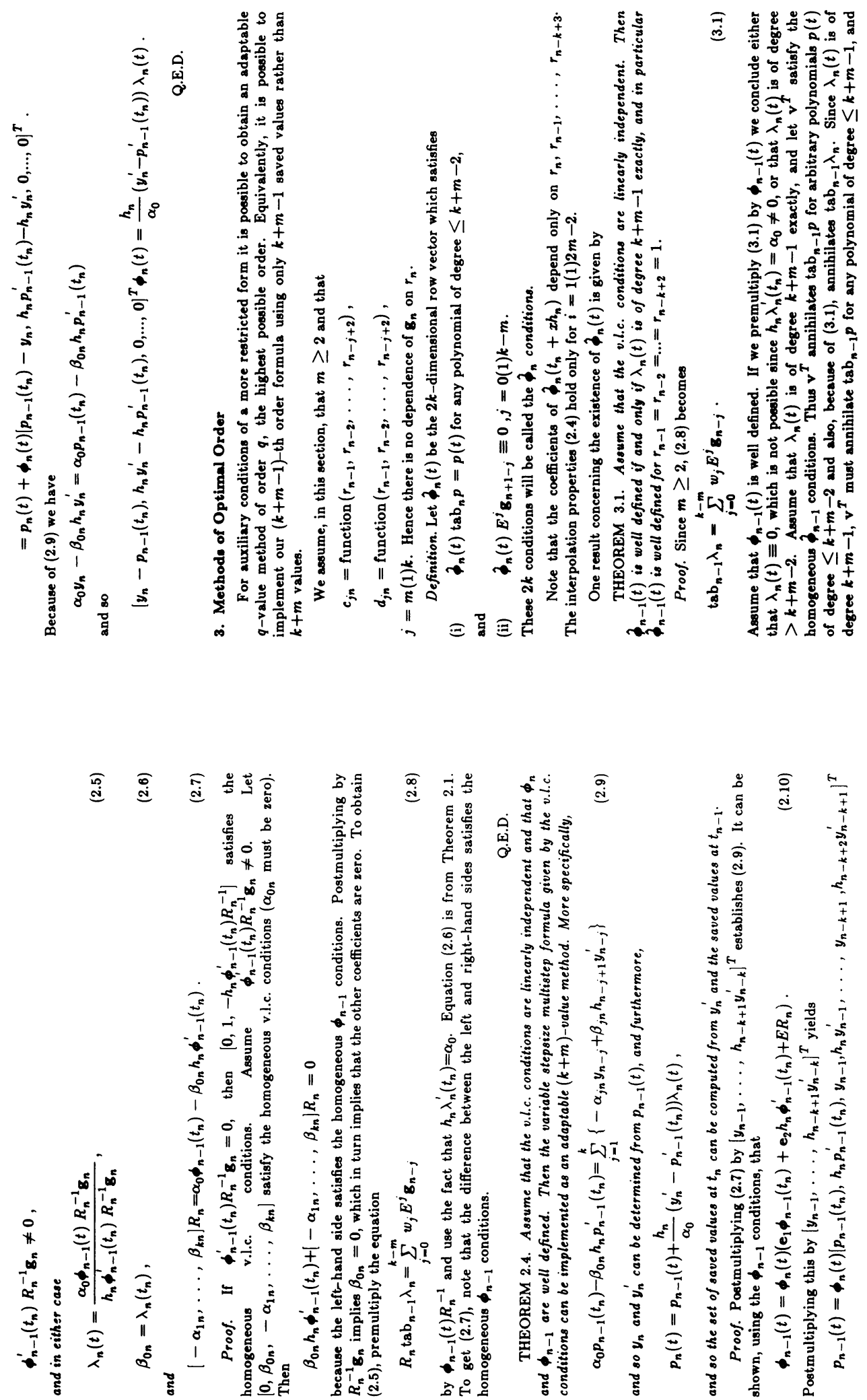

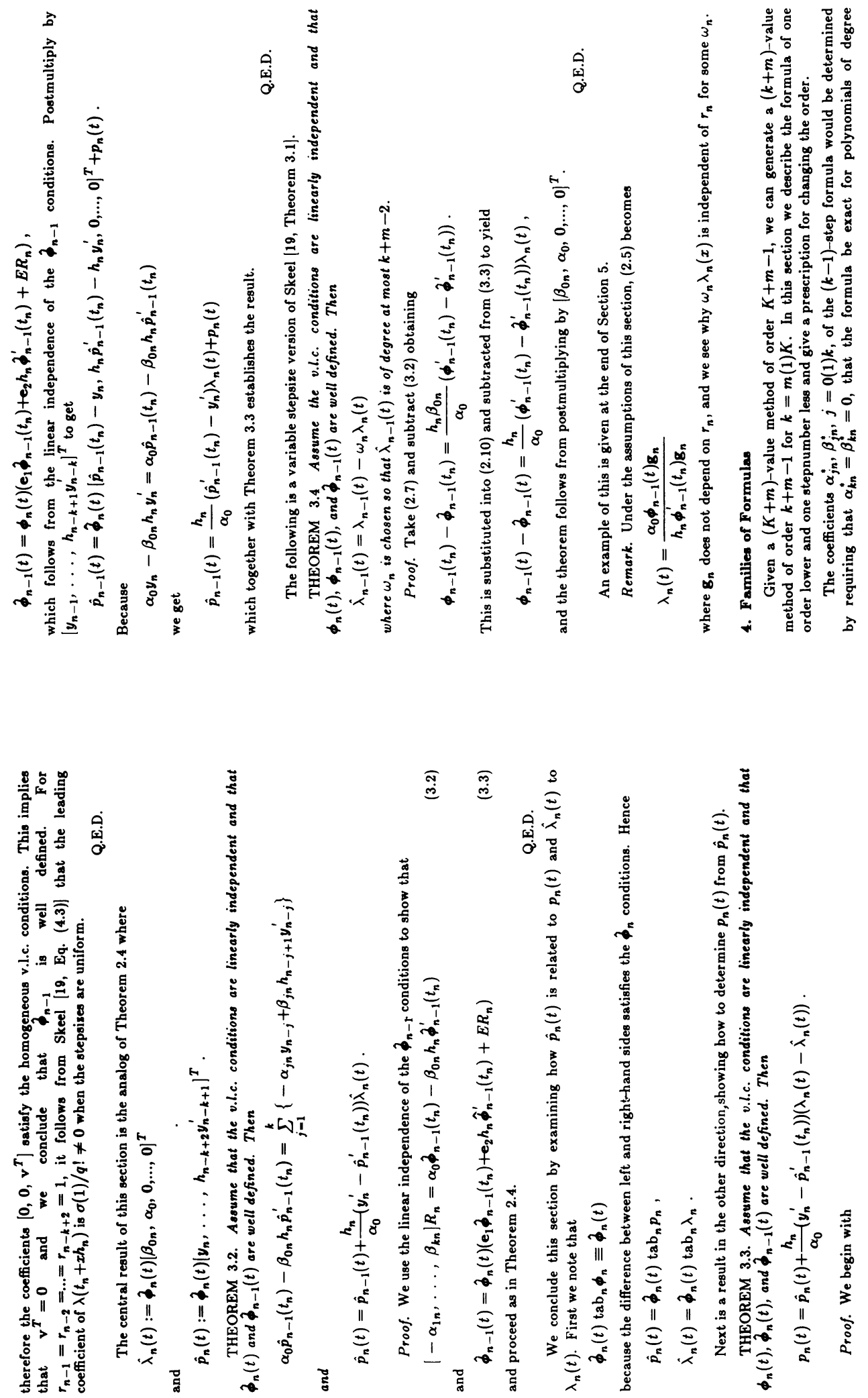

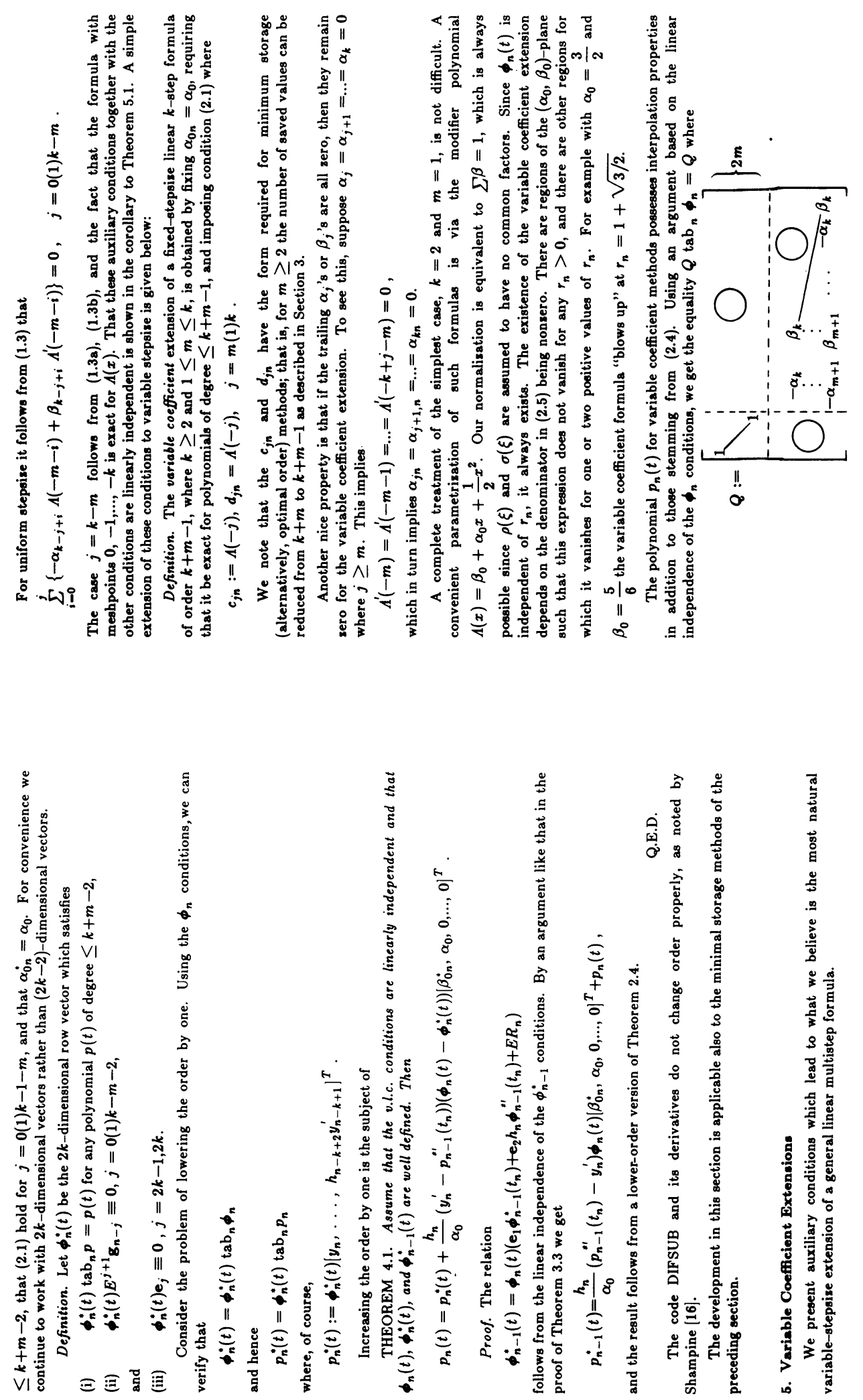

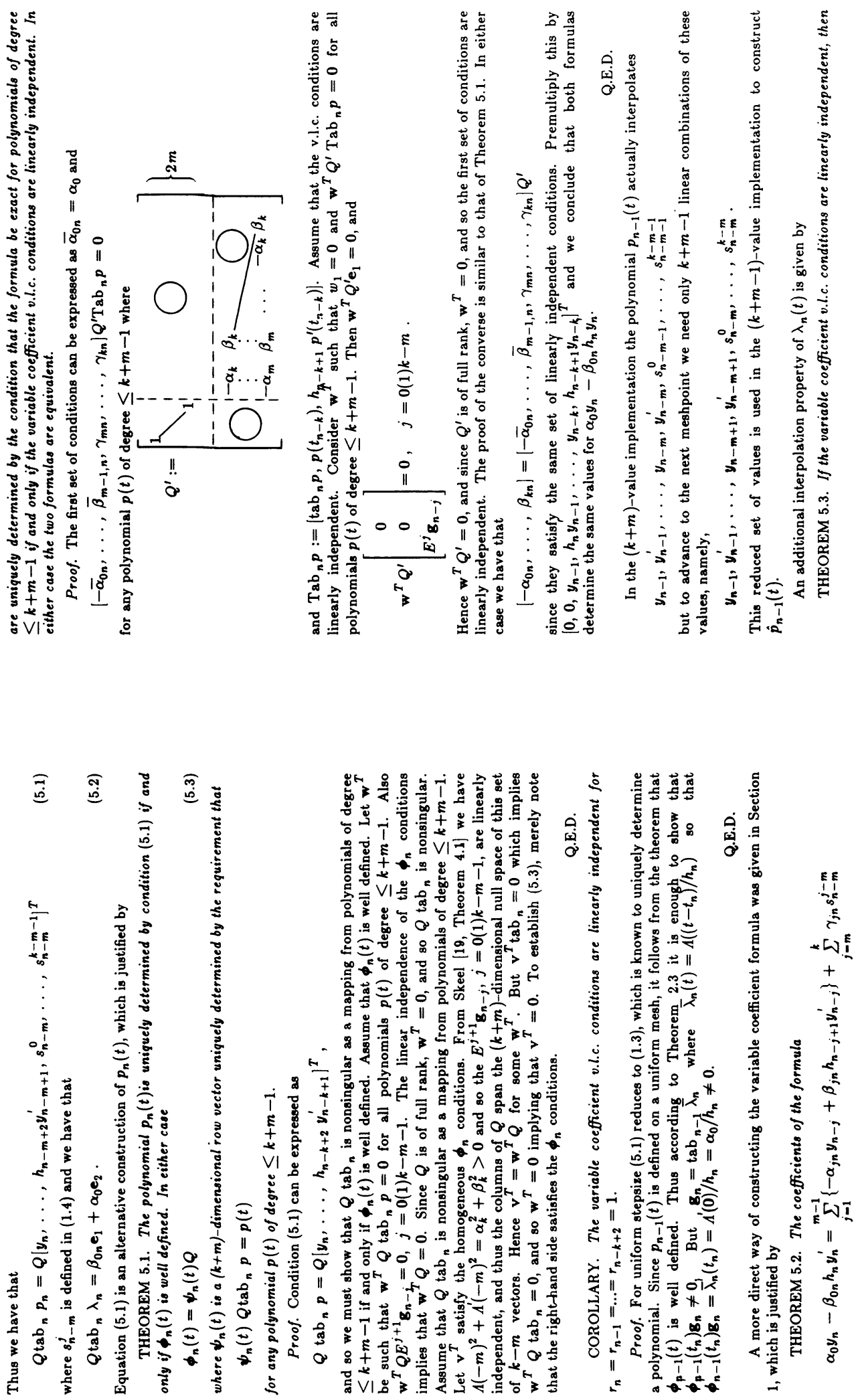

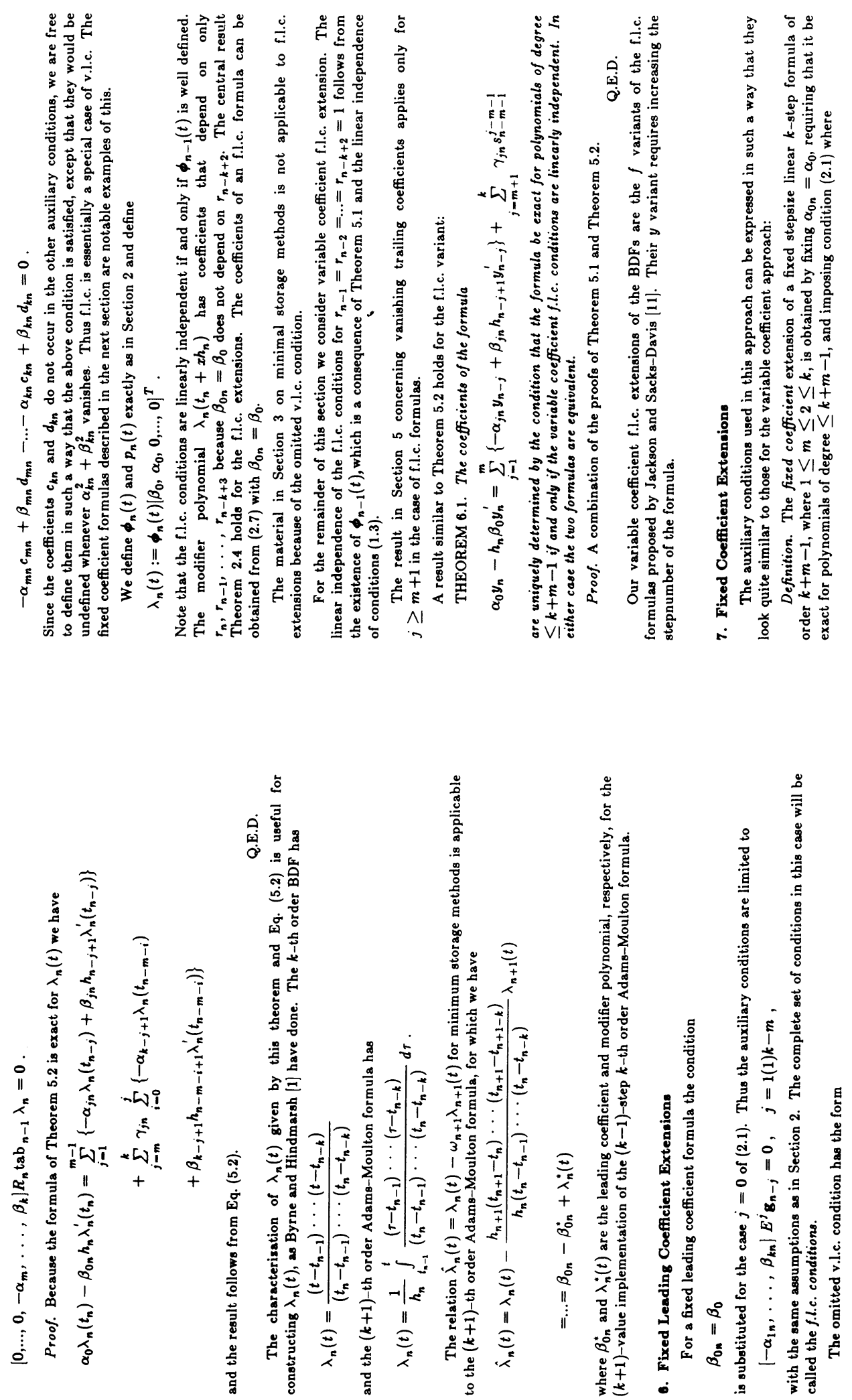

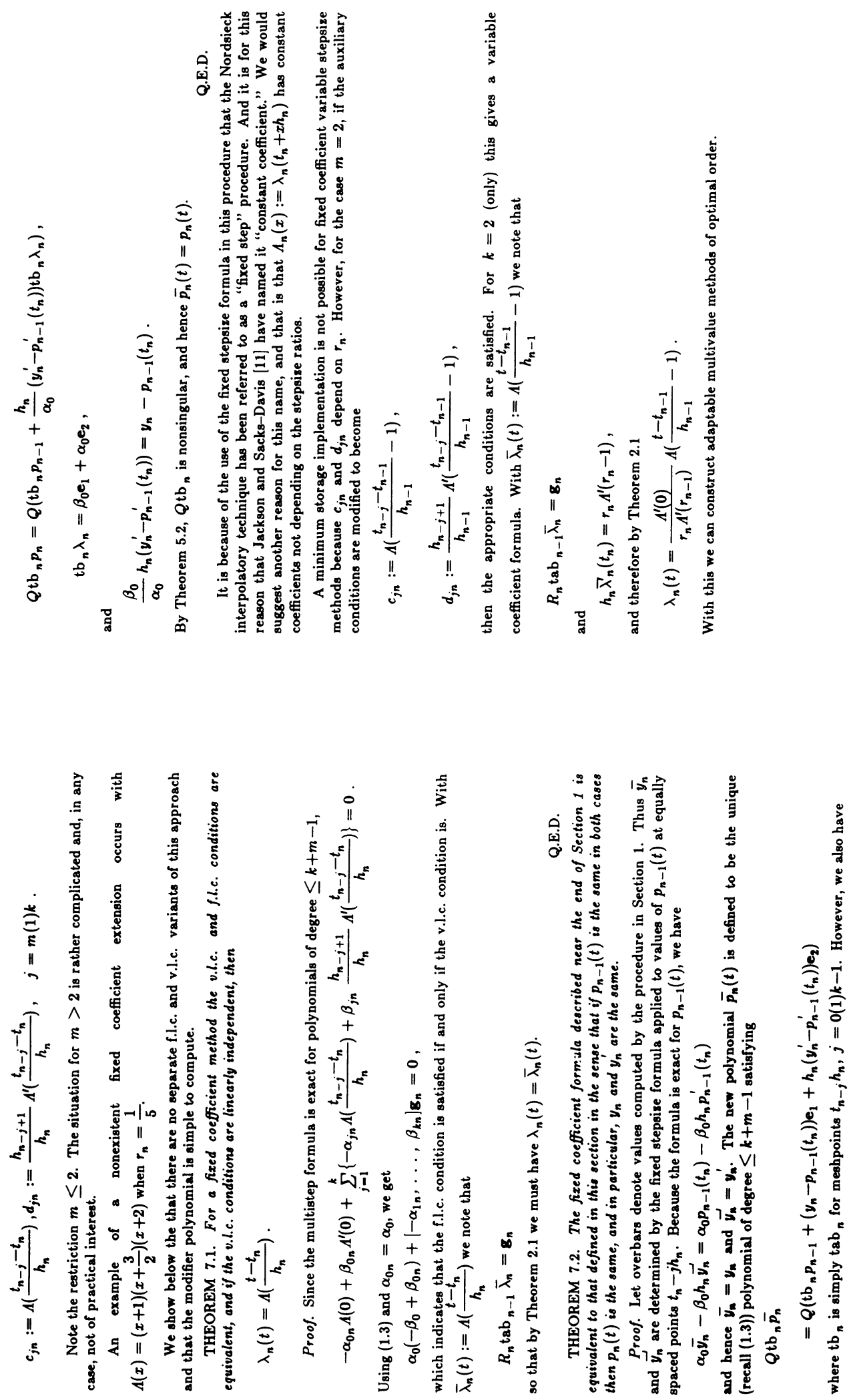